\title{
Genome-Wide Characterization of the Phosphate Starvation Response in Schizosaccharomyces pombe
}

\author{
Ian Carter-O'Connell ${ }^{1,2}$, Michael T Peel ${ }^{4}$, Dennis D Wykoff ${ }^{4^{*}}$ and Erin K O'Shea ${ }^{1,2,3^{*}}$
}

\begin{abstract}
Background: Inorganic phosphate is an essential nutrient required by organisms for growth. During phosphate starvation, Saccharomyces cerevisiae activates the phosphate signal transduction (PHO) pathway, leading to expression of the secreted acid phosphatase, PHO5. The fission yeast, Schizosaccharomyces pombe, regulates expression of the SCPHO5 homolog $\left(\right.$ pho $\left.^{+}\right)$via a non-orthologous PHO pathway involving genetically identified positive $\left(p h o 7^{+}\right)$and negative $\left(c s k 1^{+}\right)$regulators. The genes induced by phosphate limitation and the molecular mechanism by which $p h o 7^{+}$and $c s k 1^{+}$function are unknown. Here we use a combination of molecular biology, expression microarrays, and chromatin immunoprecipitation coupled with high-throughput sequencing (ChIP-Seq) to characterize the role of $\mathrm{phot}^{+}$and $\mathrm{csk}^{+}$in the $\mathrm{PHO}$ response.

Results: We define the set of genes that comprise the initial response to phosphate starvation in S. pombe. We identify a conserved PHO response that contains the SCPHO5 (pho 1+), ScPHO84 (SPBC8E4.01c), and ScGIT1 (SPBC1271.09) orthologs. We identify members of the Pho7 regulon and characterize Pho7 binding in response to phosphate-limitation and Csk1 activity. We demonstrate that activation of $\mathrm{pho}^{+}$requires Pho7 binding to a UAS in the pho $1^{+}$promoter and that Csk1 repression does not regulate Pho7 enrichment. Further, we find that Pho7-dependent activation is not limited to phosphate-starvation, as additional environmental stress response pathways require pho $^{+}$for maximal induction.
\end{abstract}

Conclusions: We provide a global analysis of the transcriptional response to phosphate limitation in S. pombe. Our results elucidate the conserved core regulon induced in response to phosphate starvation in this ascomycete distantly related to $\mathrm{S}$. cerevisiae and provide a better understanding of flexibility in environmental stress response networks.

Keywords: Phosphate starvation pathway, pho $7^{+}, \mathrm{csk}^{+}{ }^{+}$, S. pombe chlp-seq, Gene expression

\section{Background}

Inorganic phosphate $(\mathrm{Pi})$ is an essential nutrient required for signal transduction, energy metabolism, and biochemistry in all organisms. Maintaining a constant, stable concentration of internal inorganic phosphate is a major challenge for biological systems. Because external concentrations of inorganic phosphate fluctuate unpredictably,

\footnotetext{
* Correspondence: dennis.wykoff@villanova.edu; Erin_Oshea@harvard.edu ${ }^{4}$ Department of Biology, Villanova University, 800 Lancaster Ave, Villanova, PA 19085, USA

${ }^{1}$ Howard Hughes Medical Institute, Faculty of Arts and Sciences, Center for Systems Biology, Northwest Labs, Harvard University, 52 Oxford Street, Cambridge, MA 02138, USA

Full list of author information is available at the end of the article
}

microorganisms have evolved strategies to sense external phosphate levels [1-3], communicate this information to the nucleus $[4,5]$, and induce transcription to respond to phosphate flux [6-8]. The phosphate signal transduction (PHO) pathway in the budding yeast, Saccharomyces cerevisiae, is the most thoroughly studied example of phosphate homeostasis in eukaryotes [9-11].

The transcription factors Pho4 and Pho2 play a key role in the phosphate starvation response in S. cerevisiae. When cells are grown in conditions where inorganic phosphate is plentiful, Pho4 is multiply phosphorylated by the cyclin-dependent kinase-cyclin (CDK-cyclin) complex, Pho85-Pho80 [12]. When Pho4 is phosphorylated, it is localized to the cytoplasm [13,14], does not

\section{Biomed Central}


interact with Pho2 [15], and the PHO regulon is not expressed. During phosphate starvation, the CDK inhibitor Pho81 binds to the secondary metabolite myo-Dinositol heptakisphosphate $\left(\mathrm{IP}_{7}\right)$ and inhibits the Pho85Pho80 complex [16,17]. Inhibition of Pho85-Pho80 allows Pho4 to be dephosphorylated, enter the nucleus [18], co-operate with Pho2 [7], and induce a set of genes responsible for harvesting inorganic phosphate from the environment [6]. Pho4 function can be conveniently monitored by measuring the activity of the secreted acid phosphatase, Pho5, which is one of the most highly induced members of the PHO response $[19,20]$. The genes that comprise the PHO regulon have been well characterized and the precise sites within the genome where Pho4 binds during phosphate starvation are known $[7,21]$. Pho4 regulation occurs in response to changes in external phosphate levels and Pho4 activity is not thought to be regulated by other stress responses.

In this study we ask the following: is the PHO transcriptional response observed in S. cerevisiae conserved in the distantly related ascomycete, Schizosaccharomyces pombe? S. pombe presents an interesting opportunity for addressing this question because: (1) S. pombe did not experience a recent whole-genome duplication event thought to contribute to specialization [22] - possibly preventing the $\mathrm{PHO}$ response from developing a dedicated regulatory network; (2) the orthologs for the PHO pathway either do not exist (PHO81, PHO2, PHO4) or are not involved in the $\mathrm{PHO}$ response (PHO80, PHO85) in S. pombe [23]; and (3) recent work utilizing a deletion collection in $S$. pombe has outlined a basic regulatory structure for the Pi-inducible, secreted acid phosphatase pho $1^{+}$(the ortholog to PHO5) creating an opportunity for comparison with the $S$. cerevisiae $\mathrm{PHO}$ response [24]. During phosphate starvation S. pombe Pho7 - a putative transcription factor containing a $\mathrm{Zn}_{2} \mathrm{Cys}_{6}$ binuclear cluster [25] - activates $p \mathrm{hol}^{+}$expression. Csk1 - a CDK-activating kinase-activating kinase (CAKAK) [26] - represses $p h o 1^{+}$expression in high-Pi conditions. Epistasis analysis indicates that Pho7 acts downstream of Csk1.

In this study, we explore how these factors affect transcriptional output by characterizing the PHO transcriptional response in $S$. pombe. We analyze this response as a function of phosphate, Pho7, and Csk1 availability using DNA microarrays. We delineate a core PHO transcriptional response comprising the genes $\mathrm{phol}^{+}$, SPBC8E4.01c (an S. cerevisiae PHO84 ortholog), and SPBC1271.09 (an S. cerevisiae GIT1 ortholog), whose induction in response to phosphate starvation is conserved between $S$. cerevisiae and S. pombe. Interestingly, while these three genes share a functionally analogous regulatory pathway (i.e. activation through a transcription factor that is normally repressed by a kinase) we find that the mechanism for regulation differs widely between species. Our analysis of the Pho7-regulated transcriptional output - coupled with a global profile of Pho7 binding to promoters of stress responsive genes - leads us to the conclusion that, unlike Pho4, Pho7 plays a role in multiple stress response pathways. We conclude that while there is a core $\mathrm{PHO}$ transcriptional response shared between these two ascomycetes, the systems logic and specialization of PHO components varies widely.

\section{Results and discussion}

\section{pho $7^{+}$and $c s k 1^{+}$regulate a core subset of the PHO \\ response in S. Pombe}

The kinetics and maximal output of transcription vary widely between different environmental stress response pathways [27-29]. To outline the specific PHO response in $S$. pombe for subsequent analysis, and to avoid indirect activation of non-phosphate starvation regulated genes, we performed a single time-dependent, genomewide expression analysis of wild-type $S$. pombe cells in medium lacking inorganic phosphate (no-Pi conditions, see Additional file 1).

The starvation time-course revealed two distinct responses to phosphate starvation. The rapid response contained 63 genes that exhibited an increase in expression at 120 minutes post-starvation (red lines in Additional file 1, genes listed in Additional file 2; see Methods for gene selection criteria). This class contains the secreted acid-phosphatase, pho ${ }^{+}$(orthologous to ScPHO5), SPBC8E4.01c (orthologous to ScPHO84), and SPBC1271.09 (orthologous to ScGIT1). As pho $1^{+}$and SPBC8E4.01c induction has been previously observed in response to Pi starvation [24,30], we believe that this set accurately reflects the genes that respond rapidly to changes in external Pi. In contrast, the slower response (86 additional genes, blue lines in Additional file 1, genes listed in Additional file 2; see Methods for gene selection criteria) was significantly enriched for genes previously implicated in a generalized stress response [27]. We focused our attention on the fast responding genes to avoid indirect effects caused by persistent stress in cells.

Previous work indicated that $p h o 7^{+}$and $c s k 1^{+}$are important regulators of $\mathrm{phol}^{+}$expression [24]; we expected that they would also play a significant role in regulating additional components of the PHO response. To test our hypothesis, we probed the transcriptional profiles of

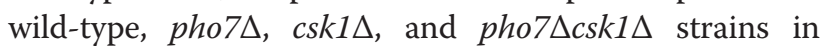
high-Pi and no-Pi conditions at 120 minutes poststarvation using DNA hybridization microarrays.

We identified 22 genes induced in response to Pi limitation (Figure 1A, first column, applying a p-value cutoff of 0.10 ; see Methods for gene selection criteria). If expression of these genes is dependent on the activity of $p h o 7^{+}$, then their transcript abundance should decrease in a pho7 $\Delta$ strain when compared to a wild-type background (both 


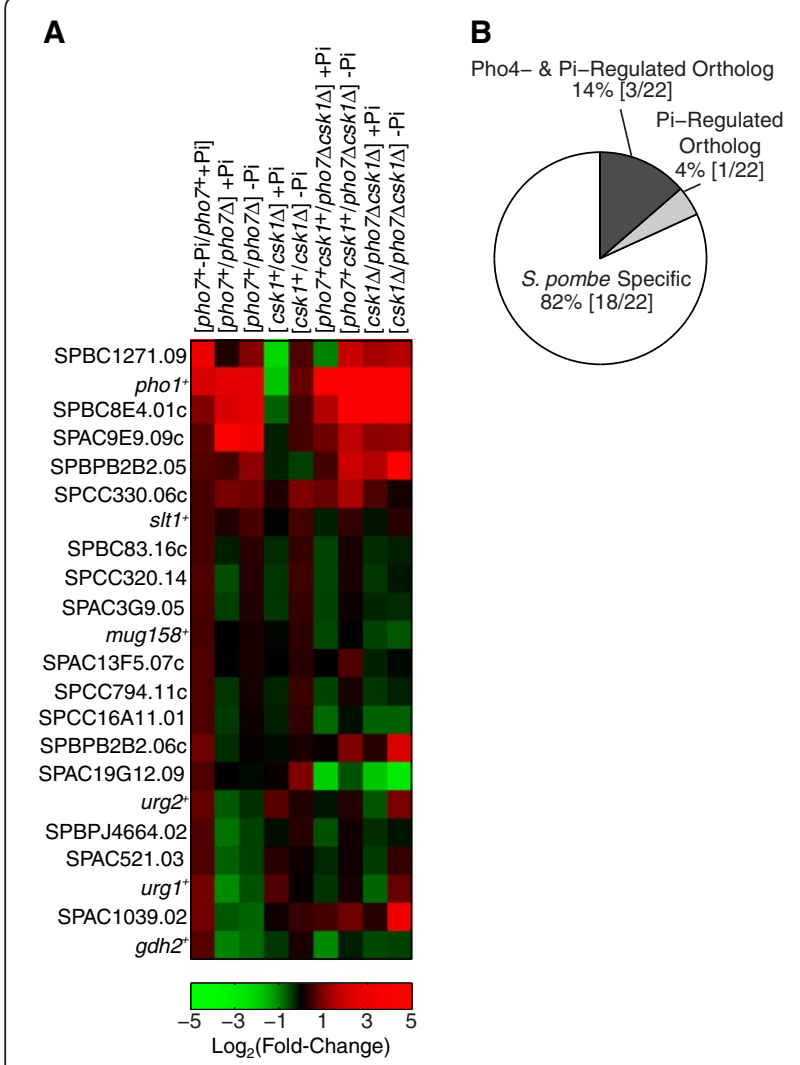

Figure $1 \mathrm{Pho7}$ and Csk1 regulate a core set of genes induced in response to phosphate limitation. (A) Heat map showing the fold change ( $\log _{2}$ scale) of Pi-starvation induced genes in a wild-type background (first column), a pho7 $\Delta$ strain in high- and no-Pi conditions vs. a pho $7^{+}$strain (second and third), a csk $1 \Delta$ strain in high- and no-Pi conditions vs. a csk1 ${ }^{+}$strain (fourth and fifth), a pho $7 \Delta \operatorname{csk} 1 \Delta$ strain in high- and no-Pi conditions vs. a pho $7^{+} \mathrm{csk} 1^{+}$ strain (sixth and seventh), and a csk1 $\Delta$ strain in high- and no-Pi conditions vs. a pho $7 \Delta \operatorname{csk} 1 \Delta$ strain (eighth and ninth) as measured by microarray analysis. Thresholds used for gene selection are described in the materials and methods. For a full list of $\mathrm{Pi}$, pho $^{+}$, and $c s k 1^{+}$dependent genes see Additional file 3. (B) Pie chart showing the percentage of genes induced in response to $\mathrm{Pi}$ limitation with Pi- and Pho4-dependent orthologs in S. cerevisiae [7].

starved of Pi). Induction of $31.8 \%(7 / 22)$ of these genes during Pi starvation is $p h o 7^{+}$dependent (Figure 1A, third column). If the repressor $\left(c s k 1^{+}\right)$prevents this induction by $p h o 7^{+}$in high-Pi conditions, then transcript abundance in a $\operatorname{csk} 1 \Delta$ strain compared to the $c s k 1^{+}$background in high-Pi conditions should increase. Three genes display this response (Figure 1A, fourth column). A complete listing of all genes regulated by $\mathrm{Pi}, p h o 7^{+}$, and/or $c s k 1^{+}$, along with their orthologs in S. cerevisiae can be found in Add-

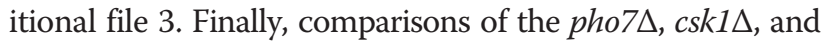
pho $7 \Delta c s k 1 \Delta$ double deletion strains confirm the previously described epistatic relationship between $p h o 7^{+}$and $c s k 1^{+}$(i. e. a loss of $p h o 7^{+}$in a csk1s switches constitutive expression to uninducible expression) (Figure 1A, last two columns).

When phosphate is limiting, S. cerevisiae Pho4, along with Pho2, induces the transcription of genes required for phosphate acquisition [7]. The orthologs of Pho4, Pho2, Pho81, and Pho80 are not found in S. pombe or involved in the $\mathrm{PHO}$ response, raising the question: does a functionally analogous signaling pathway involving $p h o 7^{+}$and $c s k 1^{+}$ regulate the PHO transcriptional response in S. pombe? Comparing the Pho4, Pi-starvation induced genes in S. cerevisiae [7] with the $p h o 7^{+}$, Pi-starvation induced genes in $S$. pombe reveals an overlap of only three orthologs (Figure 1B). For pho1 ${ }^{+}$, SPBC8E4.01c, and SPBC1271.09 a similar system of transcription factor activation, with repression by a kinase in high-Pi conditions, occurs. Unlike Pho85-Pho80 regulation of Pho4 in S. cerevisiae, most of the $p h o 7^{+}$-mediated response is independent of $c s k 1^{+}$regulation. Further, a large segment $(82 \%)$ of the observed S. pombe PHO response is not conserved between S. pombe and S. cerevisiae. Therefore, the primary role for $p h o 7^{+}$and $c s k 1^{+}$in the PHO pathway is regulating the core set of phosphate harvesting and transport genes $\left(\mathrm{phol}^{+}\right.$, SPBC8E4.01c, and SPBC1271.09).

\section{Pho7 is enriched at the $\mathrm{PHO}$ core promoters during $\mathrm{Pi}$ starvation}

Pho7 is classified as a putative transcription factor because it possesses a $\mathrm{Zn}_{2} \mathrm{Cys}_{6}$ binuclear cluster (ZC), a DNA binding domain for a number of transcription factors [31,32]. To test if Pho7 binds to $p h o 7^{+}$-regulated promoters, cells containing a functional, epitope tagged version of Pho7 (Pho7-TAP) were grown in high-Pi or no-Pi medium and purified DNA associated with $\mathrm{Pho7}$ was processed via high-throughput sequencing (ChIP-Seq, see Methods).

Surprisingly, there is widespread Pho7 binding even in high-Pi conditions (1676 peaks out of 4208 passed peak-ID thresholds; see Methods) (Additional file 4). During Pi starvation 367 Pho7-bound sites exhibit an increase in Pho7 enrichment. The highest levels of enrichment were observed in the promoters of Pho7-regulated genes identified in the microarray analysis (for a complete list of the identified peaks, see Additional file 5). Further, there is a distinct overlap between genes whose expression levels are regulated by $\mathrm{Pi}$ and/or pho $7^{+}$and those whose promoters display enrichment in Pho7 binding. 13 of the $22 \mathrm{Pi}$ dependent genes $(\mathrm{p}$-value $=8.1 \mathrm{e}-8), 16$ of the 29 pho $7^{+}$ dependent genes ( $\mathrm{p}$-value $=9.5 \mathrm{e}-9)$, and 6 of the 7 genes regulated by both $\mathrm{Pi}$ and $p h o 7^{+}(\mathrm{p}$-value $=1.3 \mathrm{e}-5)$ have promoters that are enriched for Pho7 binding in no-Pi medium ( $\mathrm{p}$-values were determined using a hypergeometric test, see Methods for details). These results are very different from the global binding profile of Pho4 in $S$. cerevisiae. In that system, Pho4 is only recruited to the 
promoters of $\mathrm{PHO}$ regulated genes during phosphate starvation and, even then, only to relatively few locations (115) within the genome [7].

Pho7 binding is significantly enriched in the promoters of $\mathrm{pho1}^{+}$, SPBC8E4.01c, SPBC1271.09, SPAC9E9.09C, SPBPB2B2.05, and SPCC330.06c in no- $\mathrm{Pi}$ conditions (Figure 2). In the case of pho1 ${ }^{+}$, SPBC8E4.01c, SPAC9E9.09C, and SPBPB2B2.05 there is enrichment of Pho7 even in high-Pi conditions (when compared to mock treated samples). This explains the previously noted basal expression of pho $^{+}$in $S$. pombe $[30,33]$ - it appears that Pho7 is bound and activating transcription even in the absence of stress (Figure 1A, second column). Based on our microarray results, we note that increased Pho7 enrichment during Pi starvation is not due to an increase in $p h o 7^{+}$transcript abundance $\left(0.05 \log _{2}\right.$ fold-change in no-Pi vs. high-Pi conditions). Moreover, we observe no significant difference in Pho7-TAP protein levels during Pi starvation (Additional file 6). Together these observations suggest that the enrichment of Pho7 in the promoters of Pho7-regulated genes is attributable to increased affinity for the promoter and not due to an increase in Pho7 abundance. Further, the number of distinct Pho7 binding events varies between different promoters (Figure 2), and we do not observe a clear correlation between the number of binding events and transcriptional up-regulation. Our attempts to identify a DNA binding motif for Pho7 based solely on our ChIP-Seq data were unsuccessful (data not shown).

\section{Csk1 does not regulate Pho7 promoter occupancy}

Motivated by the following observations, we studied what effect loss of Csk1 would have on Pho7 enrichment in high-Pi conditions: (1) Csk1 represses the expression of the core $\mathrm{PHO}$ genes (pho1 ${ }^{+}, \mathrm{SPBC} 8 \mathrm{E} 4.01 \mathrm{c}$, and SPBC1271.09) in high-Pi medium (Figure 1B, column 4); and (2) a decrease in Pi results in enrichment of Pho7 at the core PHO promoters (Figure 2). If binding of Pho7 to the PHO promoters is necessary to drive increased transcriptional output, and Csk1 represses transcription by preventing Pho7 binding, then a loss of Csk1 in high$\mathrm{Pi}$ conditions should result in an increase in Pho7 binding (mimicking the no-Pi binding profile observed for Pho7 in wild-type cells). Pho7 binding at the $p h o 1^{+}$promoter in a $c s k 1 \Delta$ background is mildly increased compared to a $c s k 1^{+}$background (Figure 3). This increase in binding is less than the observed increase in Pho7 binding in $c s k 1^{+}$cells grown in no- vs. high-Pi conditions (1.2-fold versus 4-fold), suggesting that Csk1 is not the major regulator of Pho7 binding at the pho ${ }^{+}$promoter.

We then examined the global effect of Csk1 loss on the binding profile of Pho7 using ChIP-Seq with csk1 $1 \Delta$ cells grown in high-Pi conditions. Unlike the enrichment during Pi starvation, deletion of Csk1 does not result in

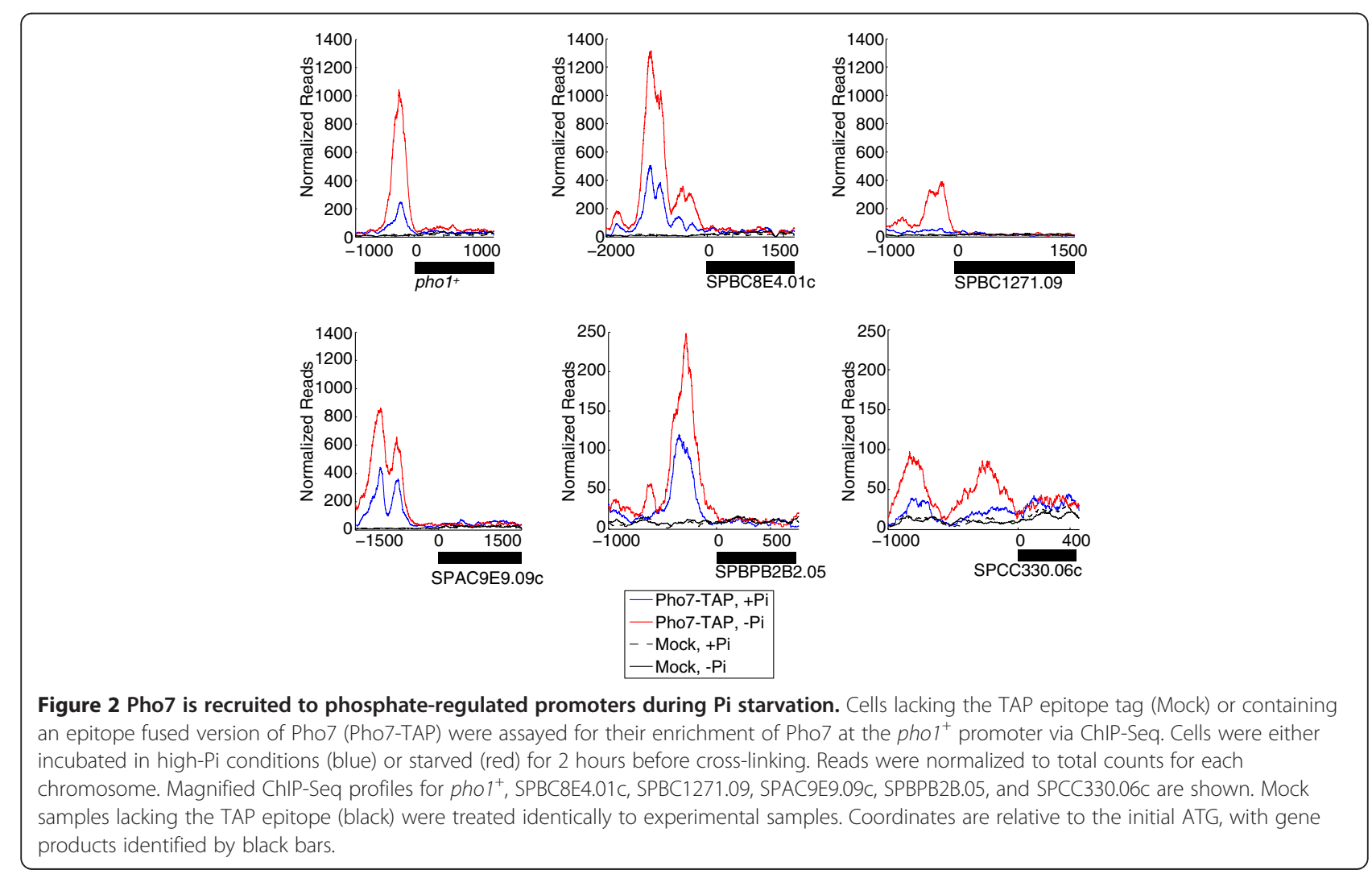



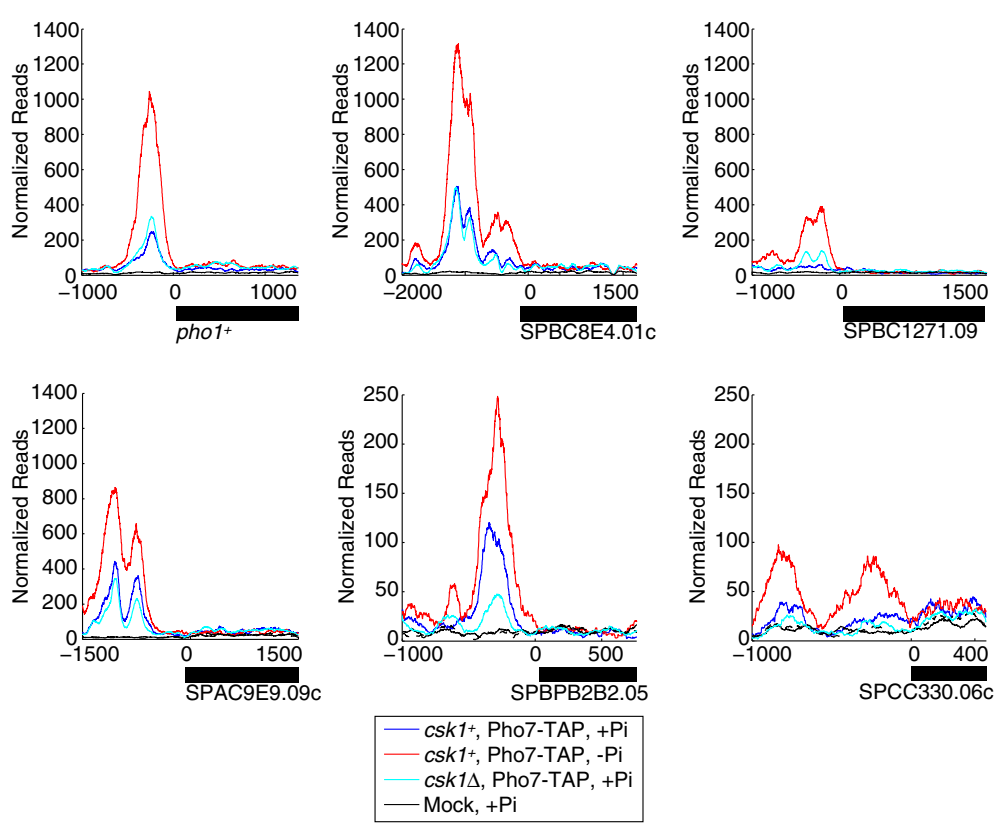

Figure 3 Csk1 does not regulate Pho7 Promoter enrichment. ChIP-Seq profiles for pho 1+, SPBC8E4.01C, SPBC1271.09, SPAC9E9.09C,

SPBPB2B.05, and SPCC330.06c are shown. Cells from either a csk $7^{+}$(blue) or csk1 $1 \Delta$ (cyan) background were incubated in high-Pi conditions for 2 hours. Mock samples lacking the TAP epitope (black) were treated identically to experimental samples. Coordinates are relative to the initial ATG, with gene products identified by black bars. Reads were normalized as previously described.

a global increase in Pho7 binding in high-Pi conditions (Additional file 7). At the core PHO responsive genes we observe either no change (SPBC8E4.01c) or a slight in-

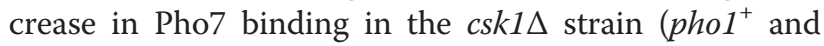
SPBC1271.09), which is still well below the enrichment seen during Pi starvation (Figure 3). As we observed during Pi starvation, the loss of Csk1 does not influence either $p h o 7^{+}$transcript abundance (-0.12 $\log _{2}$ foldchange, $\left[c s k 1 \Delta\right.$ vs. $\left.\left.c s k 1^{+}\right]+\mathrm{Pi}\right)$ or Pho7-TAP protein levels (Additional file 6). We draw two conclusions from these data: (1) the level of Pho7 bound in high-Pi conditions would be sufficient to induce high levels of transcription if not for the repressive action of Csk1; and (2) Csk1 does not repress Pho7 activity by preventing Pho7 from binding to the promoters of responsive genes.

\section{A Pho7 upstream activating sequence (UAS) and an independent $\mathrm{Pi}$ sensing module control $\mathrm{pho} 1^{+}$expression} Based on our ChIP-Seq results, we know Pho7 binds between nucleotides -280 and -180 in the $p h o 1^{+}$promoter. To determine whether the sequences in this region are necessary and/or sufficient for Pho7-dependent, Pi-starvation induced expression, we utilized an in vivo strategy for confirming Pho7-promoter interactions using exogenous expression plasmids. Briefly, differing lengths of the $p h o 1^{+}$ promoter driving the expression of yellow fluorescent protein $\left(y f p^{+}\right)$were constructed in a vector and transformed

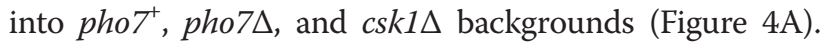
$y f p^{+}$expression was measured using FACS with mean YFP intensity serving as a proxy for promoter activity (see Methods). The $2 \mathrm{~kb}$ segment of the $p h o 1^{+}$promoter activates $y f p^{+}$expression during Pi-starvation - it exhibits a $\sim 7$-fold increase in YFP intensity upon starvation (Figure 4B). This induction is dependent on Pho7, as it is abolished in a pho7A background (Figure 4B). Surprisingly, we find that loss of Csk1 does not result in highlevels of $y f p^{+}$expression with the $2 \mathrm{~kb}$ reporter in $\operatorname{csk} 1 \Delta$ cells grown in either high-Pi or no-Pi growth media. While the levels of expression are above those seen in pho $7^{+}$cells grown in high-Pi conditions and pho7s cells grown in any condition, they are significantly lower than the levels observed in a pho7 ${ }^{+}$background in no-Pi conditions (Figure 4B). Based on our evidence that trimming the $p h o 1^{+}$promoter beyond $1 \mathrm{~kb}$ results in high level, constitutive expression of $y f p^{+}$in $c s k 1 \Delta$ cells (Figure 4D, discussed below), our results with the $2 \mathrm{~kb}$ fragment in the csk $1 \Delta$ background might be complicated by: (1) an additional repressor element located between $2 \mathrm{~kb}$ and $280 \mathrm{bp}$ in the $\mathrm{pho1}^{+}$promoter; and/or (2) differences between the chromatin structure/promoter architecture of the endogenous $p h o 1^{+}$locus and the exogenous $2 \mathrm{~kb}$ pho1 ${ }^{+} \mathrm{pr}-y f p^{+}$vector that are influencing transcription in the csk1 $1 \Delta$ cells. Therefore, we focused on the behavior of the shorter construct (280 bp pho $1^{+} \mathrm{pr}-y f p^{+}$; Figure 4D) as a proxy for the interaction of Csk 1 at the region bound by Pho7-TAP in the $p h o 1^{+}$promoter.

If the $p h o 1^{+}$promoter sequence bound by Pho7-TAP in the ChIP-Seq experiment is necessary for activation of 


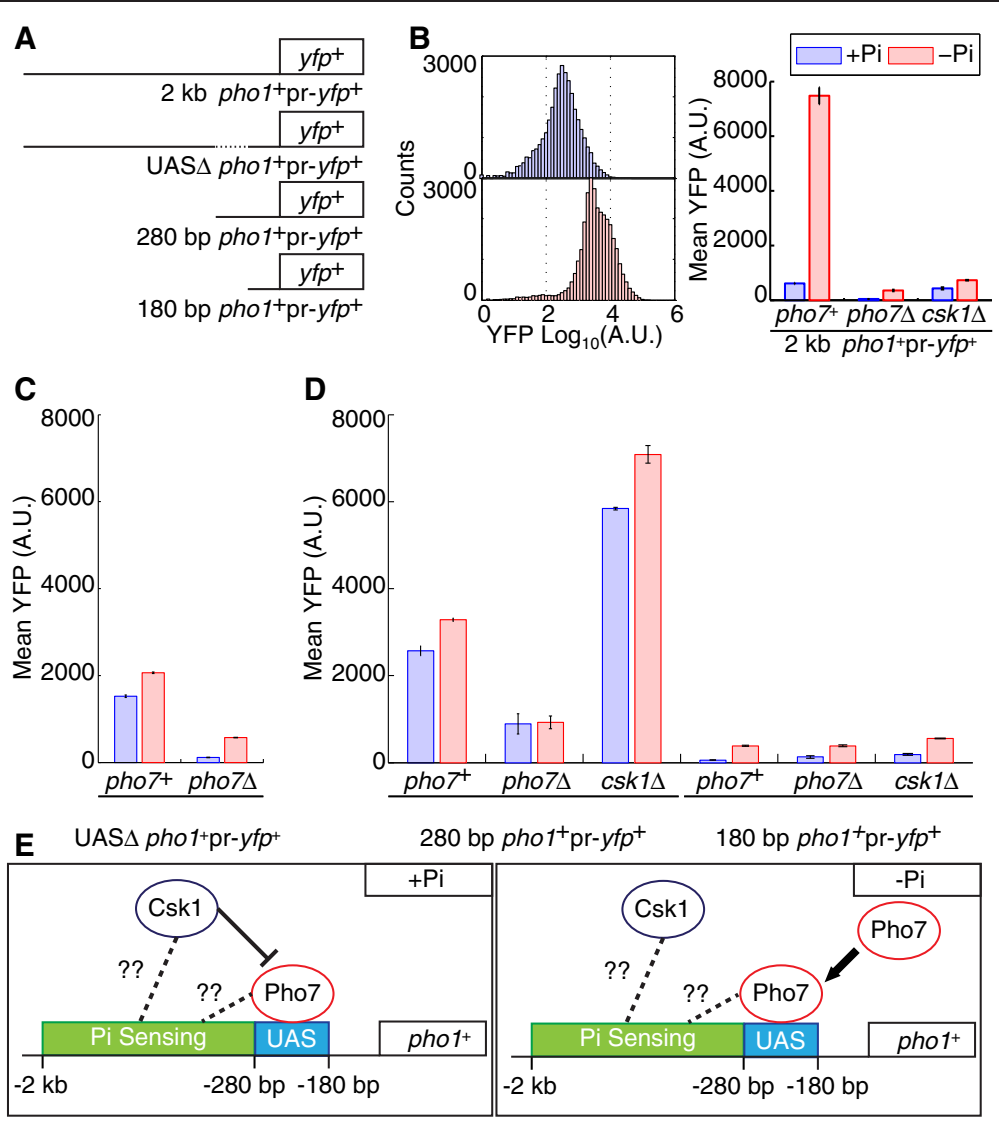

Figure 4 A Pho7 UAS in the $p h o 1^{+}$promoter is necessary expression. (A) Schematic of pho $1^{+}$promoter variants used for expression analysis.

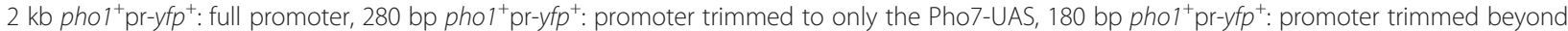

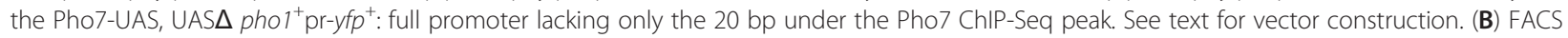

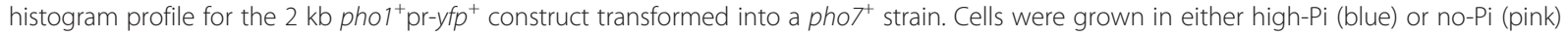
media for 4 hours prior to fixation and counting. Bar graph depicting mean YFP intensity for the $2 \mathrm{~kb}$ pho $1^{+}$pr-yff ${ }^{+}$construct transformed into a pho7 ${ }^{+}$, pho $7 \Delta$, and $c s k 1 \Delta$ strain and grown in either high-Pi (blue) or no-Pi (pink) media are given as the average of three biological replicates \pm

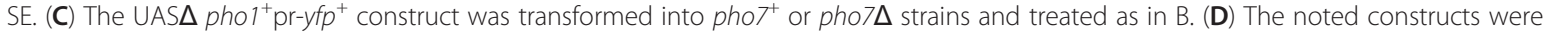

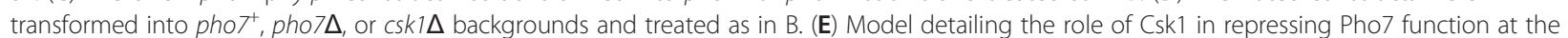
pho $1^{+}$promoter. In high-Pi conditions Pho7 is not recruited to the UAS and Csk1 interacts near the UAS to prevent full Pho7 activity. When the external concentration of Pi drops, additional Pho7 is recruited to the promoter and Csk1 repression is relieved resulting in maximal induction.

Proper regulation is controlled within a Pi sensing module located between bases -2000 and -280 in an unknown manner.

pho $1^{+}$transcription during Pi-starvation, then deletion of this region should result in a loss of $y f p^{+}$expression during Pi-starvation. To test this hypothesis, we generated a construct in which the 20 bp centered under the Pho7-TAP ChIP-Seq signal were deleted (Figure 4A,

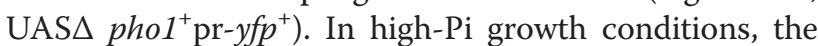
loss of the Pho7 bound region results in a slight increase in $y f p^{+}$expression (Figure $4 \mathrm{C}$, compare blue columns to the blue columns in Figure 4B), and in Pi-starvation this UAS $\Delta$ pho $1^{+}$pr- $y f p^{+}$construct no longer fully activates $y \mathrm{fp}^{+}$expression (Figure 4C, compare column 2 to column 2 in Figure 4B). The loss of $p h o 7^{+}$results in a further decrease in expression from this UASA pho $1^{+}$pr-yfp ${ }^{+}$ construct (Figure $4 \mathrm{C}$, column 4). It is possible that Pho7 recognizes additional segments of the promoter, though such contributions to activation in Pi-limiting conditions are modest. Together these results demonstrate that the Pho7-TAP bound promoter element is necessary for Pho7-dependent transcriptional activation during Pi-limitation. We have termed this region the Pho7 upstream activating sequence (UAS).

To test whether the Pho7 UAS is sufficient for Pho7dependent, Pi-limitation induced transcriptional activation we deleted all but the first $280 \mathrm{bp}$ of the $p h o 1^{+}$promoter and assayed in vivo $y \mathrm{fp}^{+}$expression (Figure 4A, $280 \mathrm{bp}$ $p h o 1^{+}$pr- $\left.y f p^{+}\right) . y f p^{+}$expression from the $280 \mathrm{bp} p h o 1^{+}$ $\mathrm{pr}-y \mathrm{fp}^{+}$construct is elevated in high-Pi conditions, and is only marginally activated during Pi-starvation (Figure 4D, pho $7^{+}$, column 1). Expression from this construct is reduced in a pho7 $\Delta$ background and is unaffected by $\mathrm{Pi}$ 
limitation - thus, expression in high-Pi conditions and the modest expression increase in Pi-limiting conditions are dependent on Pho7 (Figure 4D, pho7 , column 2). In each background tested, the mean YFP intensities from the 280 bp $p h o 1^{+}$pr- $y f p^{+}$construct vary by less than 1.5 fold between high-Pi and no-Pi conditions (Figure 4D, left panel). This is in contrast to both $y f p^{+}$expression from the $2 \mathrm{~kb}$ pho $1^{+}$pr-yff $p^{+}$construct and endogenous expression of pho $1^{+}$during Pi-starvation which exhibit $>10$-fold induction in Pi limitation. Thus, the Pho7 UAS is necessary but not sufficient for Pho7-dependent transcriptional activation during Pi-starvation.

Interestingly, the $280 \mathrm{bp} p h o 1^{+} \mathrm{pr}-y f p^{+}$construct is capable of inducing full $y f p^{+}$expression in no-Pi conditions in a csk $1 \Delta$ background (Figure 4D, csk1 $1 \Delta$, column 3). However, the $280 \mathrm{bp} p h o 1^{+} \mathrm{pr}-y f p^{+}$construct is not capable of relieving Csk1 repression in Pi-starvation conditions - expression is not induced in response to Pi limitation. Trimming beyond the Pho7 UAS results in transcriptionally inactive promoters in all backgrounds tested (Figure 4D, columns 4-6, 180 bp pho1 $1^{+}$pr-yfp ${ }^{+}$). We conclude that there must be promoter elements present in the region between $-2 \mathrm{~kb}$ and $-280 \mathrm{bp}$ in the $p h o 1^{+}$promoter that act as a Pi-sensor: (1) preventing partial Pho7-dependent activation in high-Pi conditions; and (2) are important for Csk1 de-repression during Pi-starvation.

Our FACS and ChIP-Seq results lead us to the following model for Pho7 and Csk1 regulation at the pho $1^{+}$ promoter. In high-Pi conditions some $\mathrm{Pho} 7$ is bound to the UAS in the $\mathrm{phol}^{+}$promoter. Pho7 in this state drives basal expression of $p h o 1^{+}$. Csk1, through an interaction with either Pho7 or elements near the UAS (directly or indirectly), prevents the full activation of $\mathrm{phol}^{+}$expression. The upstream Pi-sensor in the promoter ensures that Csk1 remains repressive in these conditions through an as yet unspecified mechanism. During Pi starvation, Csk1 repression is relieved and additional $\mathrm{Pho}$ is recruited to the pho $^{+}$promoter, driving maximal expression (Figure 4E). Investigating the promoter elements and transcription factors that comprise the $\mathrm{Pi}$-sensor - as well as the use of this promoter structure at additional Pho7-dependent and Pi-starvation inducible promoters - is an exciting area for future research.

\section{Pho7 regulates gene expression in response to multiple stress conditions}

During our expression analysis we noticed a set of genes with decreased expression in high phosphate conditions in a pho7 $\Delta$ background that are not induced during phosphate starvation (Additional file 3). Additionally, Pho7 is bound to the promoters of a number of these genes in the ChIP-Seq analysis (Additional file 8). These observations raise the following question: is $\mathrm{Pho7}$ dedicated solely to the phosphate starvation pathway, like Pho4, or does it play a broader role in the stress response?

To answer this question, we identified the genes from our microarray analysis that display an increase in expression between the wild-type and pho7 backgrounds (in either $+\mathrm{Pi}$ or -Pi conditions; thresholds described in materials and methods) and asked whether their promoters contained a significant peak of bound Pho7 within 800 bp of the start codon - there are 63 genes that meet these requirements. This gene set was then processed through the Gene Ontology Tools: Term Enrichment algorithm [34], allowing us to look for biological functions overrepresented in our gene set. For a GO term to be considered enriched, we required at least three unique gene products be included in the term family and the enrichment must have a $\mathrm{p}$-value $\leq 0.01 .32$ of the original 63 genes meet these requirements and are classified by the highest parent term available (Figure 5A). 15 of these 32 genes are involved in transmembrane transport, including 3 involved in phosphate ion transport. Characterizing the transmembrane category in further depth we find genes involved in iron, copper, and zinc transport (Figure 5B). A complete listing of GO Terms and the enriched gene set can be found in Additional file 9 .

From the 32 identified genes, we utilized $g p d 1^{+}, h x k 2^{+}$, $\mathrm{fiol}^{+}$, and $\mathrm{ctr}^{+}$expression as proxies for Pho7-mediated transcriptional induction in various stress conditions. Gpd1 is a glycerol-3-phosphate dehydrogenase that synthesizes glycerol and is essential for survival during osmotic stress [35]. During osmotic stress, glycerol pools increase, protecting the cell. Hxk2 is a hexokinase that plays a role in regulating alternative carbon utilization when glucose sources are limited [36]. It is maximally induced in response to a switch from glucose to glycerol as a carbon source. Fio1, in conjunction with Fip1, comprises the oxidase-permease iron transport system responsible for harvesting iron in depleted conditions [37]. During iron repletion fio $^{+}$is repressed by the activity of Fep1, an iron sensing transcription factor [38]. During iron starvation $\mathrm{fiol}^{+}$is de-repressed and induced $\sim 70$-fold. Finally, Ctr4 is a high-affinity copper transporter that is induced in copper depletion conditions by Cuf1, a copper sensing transcription factor [39]. We designed RT-qPCR primer sets for each of these genes and measured their expression as a function of osmotic, iron, copper, and carbon utilization stress in both $p h o 7^{+}$ and pho7 $\Delta$ backgrounds (see Methods).

As previously demonstrated [24], loss of Pho7 completely abrogates induction of $\mathrm{phol}^{+}$in no-Pi medium (Figure 5C, first panel). For each of the additional stresses tested, the loss of Pho7 causes a significant decrease (p-value $\leq 0.05)$ in the maximal induction of the target gene (Figure 5C). The Pho7 dependence of these genes varies, with some $\left(\mathrm{fiol}^{+}, \mathrm{ctr} 4^{+}\right)$showing a relatively minor 

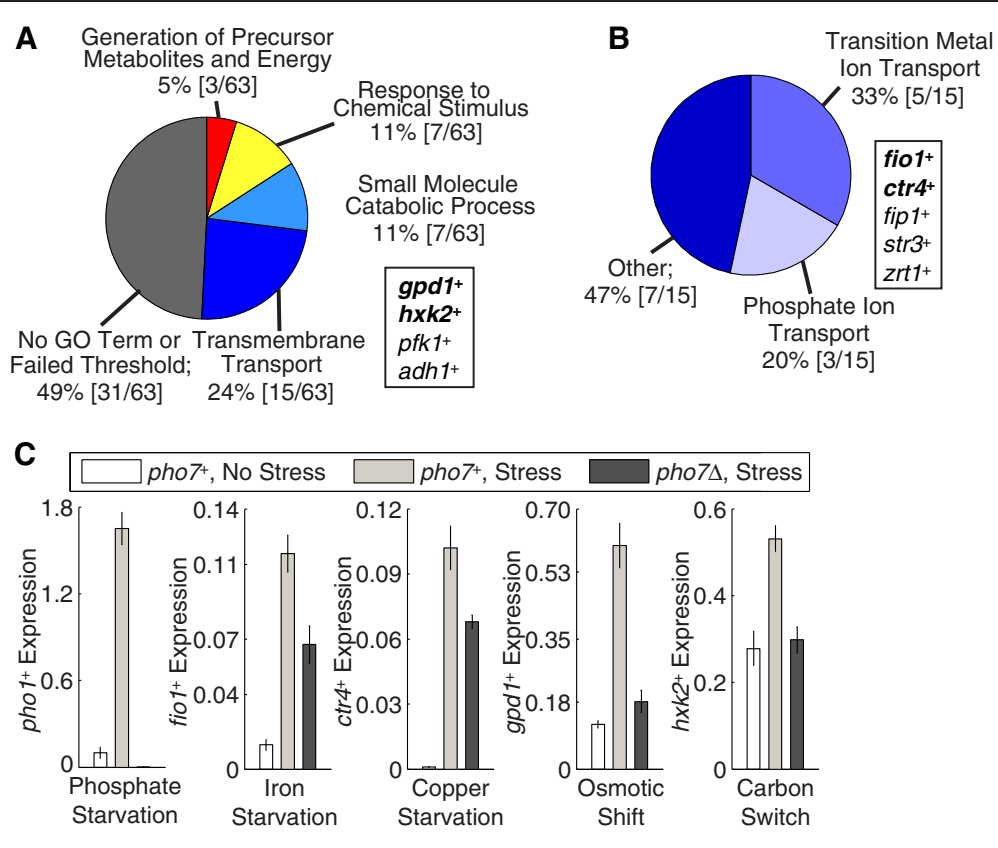

D

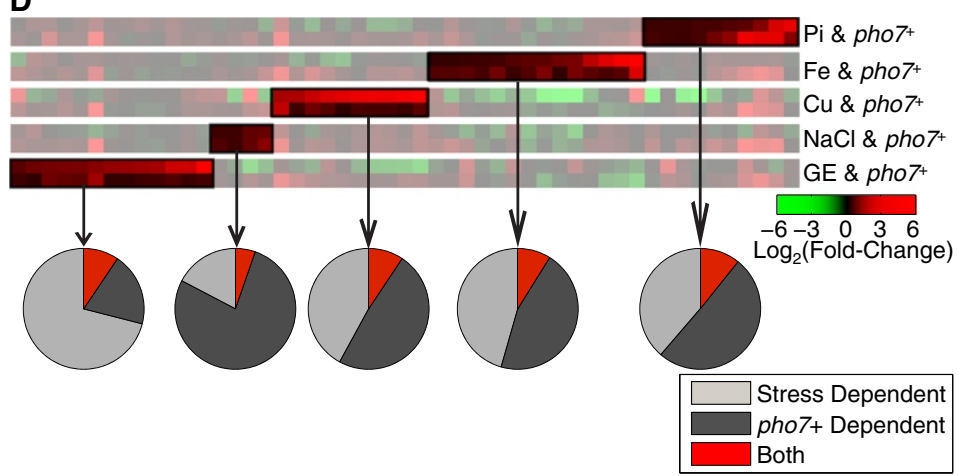

Figure 5 pho $^{+}$functions in additional stress responses. (A) Genes with a Pi- and pho $7^{+}$-component based on microarray analysis were crossreferenced with a list of genes with pho $7^{+}$enrichment within $800 \mathrm{bp}$ of the initial ATG (ChIP-Seq). The pie chart shows the GO Term enrichment for the identified genes as compared to a background model of all S. pombe transcripts. The boxed inset highlights a number of genes that were found in the small molecule catabolic process class, with those in bold serving as the initial targets for expression analysis. P-values for the enrichment against the background model are given. (B) The transmembrane transport category from A was expanded to identify specific ions whose transport is regulated by pho $7^{+}$. Bold candidate genes served as initial target for expression analysis. (C) pho $7^{+}$cells were incubated in either normal (white) or stress (light gray) conditions, and the expression of the appropriate gene was measured by RT-qPCR. pho7 $\Delta$ cells were also stressed (dark grey) to identify the level of $p h o 7^{+}$-dependent regulation. Expression was normalized to act $1^{+}$. Shown is the average of three biological replicates \pm SE. (D) Heat map displaying the fold-change $\left(\log _{2}\right.$ scale) for genes induced during stress in a pho $7^{+}$-dependent manner. The top track in each sub-column compares expression in replete versus stress conditions for wild-type cells; the bottom track compares pho7 ${ }^{+}$ to pho7 $\Delta$ cells in the displayed stress (Pi: phosphate starvation, Fe: iron starvation, Cu: copper starvation, NaCl: osmotic shift, GE: carbon switch). Boxed regions identify genes that are induced in response to stress and require pho $7^{+}$for their stress response. Pie charts depict the percentage of genes responding to stress in a pho $7^{+}$independent manner (light grey), repressed by the loss of pho $7^{+}$but unaffected by stress (dark grey), and dependent on both (red).

Pho7 component, while others $\left(g p d 1^{+}, h x k 2^{+}\right)$appear fully dependent on $\mathrm{Pho} 7$ to reach a peak level of induction in stress. None of the Pho7-regulated responses were as dramatic as that observed for Pi starvation, which may indicate that Pho7 plays a more subtle role in coordinating expression at Pi-independent loci. Given this subtle response, the only recent availability of a deletion collection for S. pombe [40], and the fact that Pho7 function was unverified until recently, it is not surprising that the more general role for Pho7 has not been previously observed.

It remains possible that the stress response effects we observe are artifacts limited to only the few genes we studied using RT-qPCR. Using microarray analysis with RNA collected from $p h o 7^{+}$or pho7 4 cells grown in nonstress and stress conditions, we examined the stress response mediated by $p h o 7^{+}$in the above conditions. 
We find that of 274 genes induced in the stress conditions studied, 44 genes are $p h o 7^{+}$dependent $(7$ genes are induced in multiple stress conditions) (Figure 5D and Additional file 10). $p h o 7^{+}$is responsible for coordinating between $11.7-23.5 \%$ of the total stress response in each condition (for comparison, $p h o 7^{+}$coordinates $21.7 \%$ of the Pi starvation response using these thresholds). In each stress we find enrichment of distinct GO terms. The set of iron-responsive, $p h o 7^{+}$-regulated genes is significantly enriched for the biological process of iron assimilation $\left(\right.$ fio $^{+}$, fip $^{+}$, str $^{+}$, sib2 $2^{+}, \mathrm{p}$-value $\left.=5.2 \mathrm{e}-09\right)$, the set of copper-responsive, $p h o 7^{+}$-regulated genes is enriched for copper ion transport $\left(c t r 4^{+}\right.$and $c t r 5^{+}$, p-value $\left.=5.6 \mathrm{e}-05\right)$, and the osmotic shock-responsive, $p h o 7^{+}$-regulated gene set is enriched for metal ion transport $\left(z r t 1^{+}\right.$and $c t r 4^{+}$, $\mathrm{p}$-value $=7.8 \mathrm{e}-04)$. With the carbon switch, $p h o 7^{+}$-regulated response we see an enrichment of genes responsible for small molecule metabolism as well as conjugation. The reasoning for the conjugation process enrichment or why pho $7^{+}$would be involved is unclear. Overall, the biological processes are generally linked with transmembrane transport, suggesting that $p h o 7^{+}$is responsible for coordinating the correct transport of nutrients required for each stress response. Unlike the system in S. cerevisiae, where the central Pi-starvation regulator is tightly linked with the $\mathrm{PHO}$ response, the $p h o 7^{+}$based system in S. pombe functions differentially in a number of stress response networks.

\section{Conclusions}

In this study we have defined and characterized the gene regulatory network in $S$. pombe responsible for coordinating the response to inorganic phosphate starvation. There are two distinct temporal responses in the PHO pathway in S. pombe: a fast response concerned with immediately harvesting inorganic phosphate from the environment and transporting it into the cell, and a slower one associated with a general stress response. Within the fast response we define a core $\mathrm{PHO}$ regulon comprised of the pho1 ${ }^{+}$, SPBC8E4.01c, and SPBC1271.09 genes whose induction in response to phosphate starvation, and regulatory behavior, has been conserved between S. pombe and S. cerevisiae.

Unlike the PHO response in S. cerevisiae, however, the positive regulator in $S$. pombe is bound to the $p h o 1^{+}$and SPBC8E4.01c promoters irrespective of external phosphate availability. Using our Pho7-TAP ChIP-Seq dataset, we identified a single Pho7 binding region in the pho $1^{+}$promoter. This region - located between nucleotides -265 and -245 - serves as a Pho7-dependent UAS, which is necessary for transcriptional activation of $p h o 1^{+}$. The interaction of Pho7 with the UAS leads to a basal expression of the secreted acid phosphatase in high-Pi conditions. The $1.8 \mathrm{~kb}$ region preceding the UAS in the pho $1^{+}$promoter is required for $\mathrm{Pi}$ sensing, coordinating the activation by Pho7 and repression by Csk1 based on $\mathrm{Pi}$ availability. Csk1 prevents full activation of Pho7 during phosphate replete conditions and repression is maintained even in the minimal UAS construct (280 bp pho1 $1^{+} p r-y f p^{+}$). During phosphate starvation this inhibition is relieved (through an unknown mechanism) and additional Pho7 is recruited to a number of sites throughout the genome, causing further induction of $p h o 7^{+}$-dependent genes (see Figure 6 for a comparison of the S. cerevisiae and S. pombe PHO pathways). In previous work, Csk1 was shown to regulate transcription by activating the positive transcription elongation factor b (P-TEFb) ortholog, Cdk9 [41]. Cdk9 coordinates transcript elongation and processing, and its full activation by Csk1 leads to an increase in CTD kinase activity [42]. How this generally positive regulatory network is switched to an inhibitory role in the PHO system remains an open question.

We were also surprised to find that Pho7 was bound throughout the genome in both high-Pi and no-Pi conditions. We had thought based on previous evidence that Pho7, like Pho4, would be specific to the PHO response. Instead we demonstrate that Pho7 binds within the promoters of additional stress responsive genes and plays a role in iron, copper, osmotic, and alternative carbon utilization stress. Each stressor elicits a different pho7 ${ }^{+}$-dependent transcriptional response, though it appears that the main regulatory role of $p h o 7^{+}$is coordinating stress-specific transmembrane transport. There must exist some mechanism to either direct Pho7 to the proper location for inducing the correct genes or activate Pho7 at only the appropriate locations (or some mixture of both). In S. cerevisiae, the osmotic, oxidative, and glucose limitation stress responses are mediated by the transcription factor Msn2 [43]. In normal conditions, Msn2 is phosphorylated and its entry into the nucleus is limited [44]. Different stresses elicit distinct dynamics of nuclear transport, leading to different transcriptional outputs [29]. Given that Pho7 is bound to the genome constitutively, we do not expect that nuclear exclusion will play as large a role as it does with Msn2 regulation, but it remains possible that differential post-translational modifications are responsible for this combinatorial activation by Pho7. Pho7 may be playing a more passive role in regulation, with additional factors determining Pho7 genomic localization.

Nonetheless, we have demonstrated that within the evolutionary parallel signal transduction networks that comprise the $\mathrm{PHO}$ pathway there exists a core $\mathrm{PHO}$ transcriptional regulon. The specific mechanisms involved in regulating the $\mathrm{PHO}$ response in S. cerevisiae and S. pombe show remarkable flexibility. An interesting area for future research centers on the environmental factors that contributed to the development of these two parallel networks. Why is the PHO response in S. pombe 


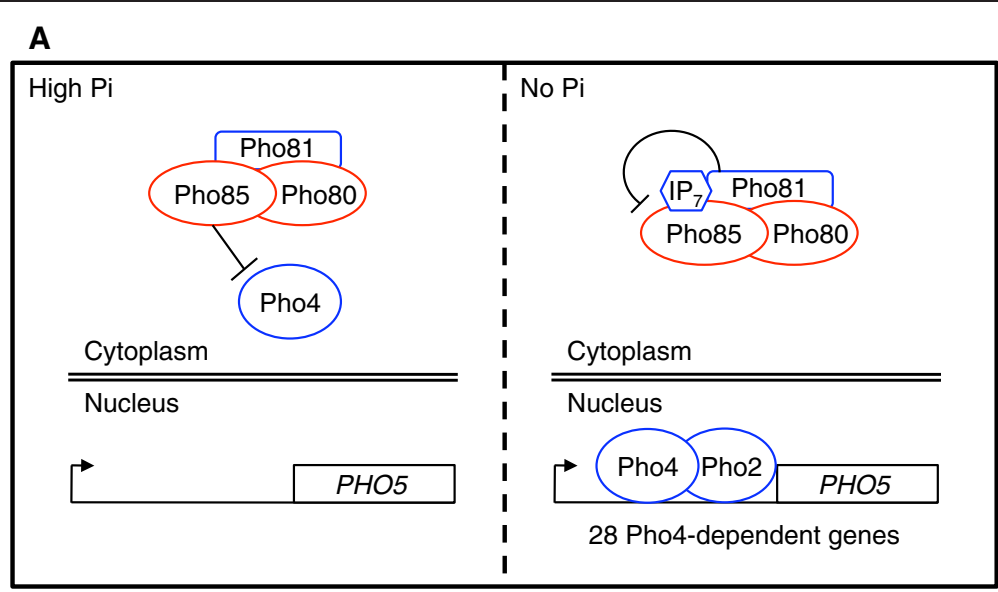

B

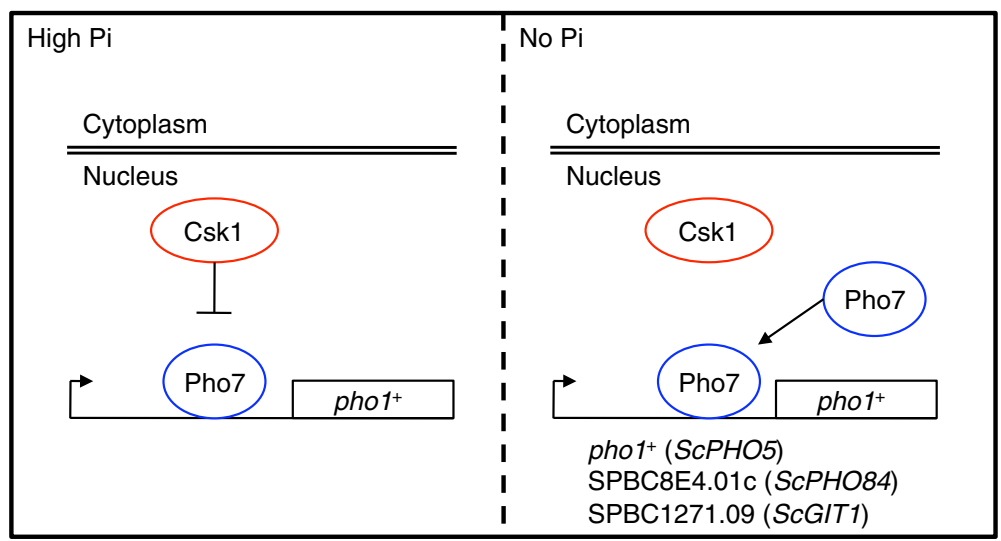

Figure 6 A comparison of the S. cerevisiae and S.pombe PHO signaling pathways. (A) A schematic depicting the PHO signaling pathway in S. cerevisiae. In conditions where Pi is plentiful (high Pi, left) Pho4 is multiply phosphorylated by the Pho85-Pho80 complex and is excluded from the nucleus. Depletion of inorganic phosphate (no Pi, right) leads to the IP7-Pho81-dependent inhibition of Pho85-Pho80. Relief of Pho85-Pho80 mediated repression allows unphosphorylated Pho4 to enter the nucleus, interact with Pho2 at PHO-specific genes, and induce transcription. (B) A schematic depicting the PHO signaling pathway in S. pombe. In high Pi conditions (left) some Pho7 is bound to the promoters of Pi- and pho $7^{+}$-dependent genes. Bound Pho7 is prevented from reaching maximal activation by the repressive effects of Csk1. Loss of external phosphate (right) results in the recruitment of additional Pho7 to the promoter and the relief of Csk1-mediated repression. The Pho7-dependent genes identified in this study that are regulated in this manner are listed (S. cerevisiae orthologs in parenthesis).

under the control of a general stress transcription factor, Pho7, while S. cerevisiae has developed the phosphate starvation specific pathway for Pho4 activation? What are the environmental pressures that favor a "leaky" response in $S$. pombe and a tightly controlled one in $S$. cerevisiae? Broadly speaking, our study provides a framework for determining the fundamental requirements for regulating phosphate homeostasis in Ascomycota and the specific points in the signal transduction pathway that can be altered as conditions merit.

\section{Methods}

\section{Growth conditions and strains}

$S$. pombe cells were maintained in previously described YES or EMM media [45]. The yeast strains used were: DP1 (972 $\left.h^{-}\right)$, DP18 (ura4-D18 ade leu $^{+} h^{+}$), DP81 (pho7DKANMX6 $972 h^{-}$), DP94 (Pho7-TAPKANMX6 $\left.972 h^{-}\right), \quad$ DP106 (csk1 $\left.\triangle N A T M X 6972 h^{-}\right), \quad$ DP109 (csk1 KKANMX6 ura4-D18 ade leu $\left.^{+} h^{+}\right)$, DP111 (pho7DKANMX6 ura4-D18 ade $^{+} \mathrm{leu}^{+} h^{+}$), DP113

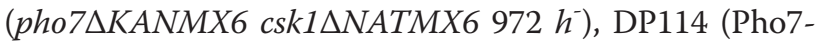
TAPKANMX6 csk1 1 NATMX6 ura4 $972 h^{-}$), and DP115 (Pho7-TAPKANMX6 csk1 $\triangle N A T M X 6$ ura4 $972 h^{+}$). The functionality of the Pho7-TAP allele was confirmed by both liquid phosphatase assay and RT-qPCR analysis and it behaves as $p h o 7^{+}$. To tag Pho7 and delete $c s k 1^{+}$ we utilized a PCR fragment containing the marker of interest flanked by homologous regions for the specific gene target. Cells were transformed with lithium acetate and polyethylene glycol 8000 [45]. Primers used for deletion or tagging are found in Additional file 11. To provide consistency with previously published results for inorganic phosphate starvation, all starvation experiments were conducted with cells incubated in a 
90\%SD-10\%EMM media, which has been previously described [24].

\section{Microarray analysis and data processing}

Strains were grown in $90 \%$ SD-10\%EMM medium con-

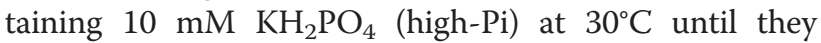
reached early-log phase $\left(\mathrm{OD}_{600}=0.15-0.25\right)$. Cells were collected via filtration, washed twice, transferred to fresh media lacking $\mathrm{Pi}$ (no-Pi), and grown at $30^{\circ} \mathrm{C}$ for up to 4 hours. Immediately prior to starvation $(\mathrm{t}=0), 20 \mathrm{~mL}$ of cells were added to $30 \mathrm{~mL}$ of methanol kept at $-65^{\circ} \mathrm{C}$ to prevent further transcription or RNA degradation. At $30,60,120$, and 240 minutes post-starvation this process was repeated. Cells were left in methanol for 10 minutes, pelleted, washed quickly in autoclaved water, resuspended in $750 \mathrm{uL}$ of RNAlater (Ambion), and snapfrozen in liquid nitrogen. RNA was extracted using the RNeasy Mini kit (Qiagen). cDNA was generated in a reverse transcriptase reaction using $10 \mu \mathrm{g}$ total RNA with a 1:1 mixture of oligo-dT and random hexamer primers (Operon) and a 2:3 ratio of amino-allyl-dUTP:dTTP (Sigma). Superscript II RT (Invitrogen) was added and the reaction mixture was incubated at $42^{\circ} \mathrm{C}$ for 2.5 hours. cDNA was purified using a PCR purification kit (Qiagen) after completing hydrolysis of remaining RNA. An equal amount of cDNA from each time point was pooled to provide the reference sample. Purified cDNA samples were labeled using $\mathrm{N}$-hydroxyl succimamide esters of either Cy3 or Cy5 dyes (GE Biosciences). 300 ng of the Cy3 (each individual time point) and $300 \mathrm{ng}$ of the Cy5 (pooled reference) labeled sample was competitively hybridized to custom Agilent $8 \times 15 \mathrm{~K}$ S. pombe twocolor expression microarrays (GEO Platform:GPL15827) in 2xGEx Hybridization Buffer (Hi-RPM) (Agilent) for 17 hours at $60^{\circ} \mathrm{C}$. Microarrays were washed and immediately scanned using an Axon 4000B scanner [46]. The mean intensity of each spot in the Cy3/Cy5 channels was extracted using the GenePix 5.1 software, followed by lowess and quantile normalization performed with the MATLAB bioinformatics toolbox. Expression ratios for each time point, $\mathrm{x}$, were normalized to $\mathrm{t}=0\left(\log _{2}\left[\mathrm{Cy} 3_{\mathrm{t}=\mathrm{x}} / \mathrm{Cy} 5_{\text {pool }}\right]-\right.$ $\left.\log _{2}\left[\mathrm{Cy} 3_{\mathrm{t}=\mathrm{O}} / \mathrm{Cy} 5_{\text {pool }}\right]\right)$ and thresholds for induced genes were set at $\geq 2 \sigma+$ median $\log _{2}$ fold change for each time point (1.00 $\log _{2}$ fold change at 120 minutes, $1.24 \log _{2}$ fold change at 240 minutes). Genes above threshold at both 120 minutes and 240 minutes post-starvation were classified as the rapid response. Genes above only the 240-minute threshold were classified as the slow response. The starvation time course was not repeated. Results for all of the microarray experiments conducted in this study are available through NCBI-GEO [GEO:GSE39478].

To determine the extent of $p h o 7^{+}$and $c s k 1^{+}$regulation within the PHO response we grew the relevant strains (DP1, DP81, DP106, DP113) as described above, with the exception that cells were split into either high-Pi or no-Pi media and grown for 2 hours prior to RNA collection. Two independent biological replicates were performed for each of the conditions tested except for the pho $7^{+}$csk $1 \Delta /$ pho $7 \Delta \operatorname{csk} 1 \Delta$ comparison in no-Pi media. For each of these arrays the two probes used to detect each ORF were averaged and treated as single data points with p-values determined using a student's t-test with a one-tailed distribution against the null hypothesis in the MATLAB software. Thresholds were set at $\geq 1.8$ $\log _{2}$ fold-change to facilitate comparison with the previously characterized S. cerevisiae data set [7]. Genes passing the induction threshold also had to pass a p-value threshold of $\leq 0.10$. Clustering analysis was completed using k-means clustering in the Cluster 3.0 program [47] after empirically determining the optimal number of clusters using the MATLAB bioinformatics toolbox.

\section{Chromatin immunoprecipitation of Pho7-TAP with high-throughput sequencing (ChIP-Seq)}

ChIP-Seq was performed on the DP1, DP94, and DP115 strains as previously described [7,48-50]. Cells were grown to early log-phase $\left(\mathrm{OD}_{600} \sim 0.18\right)$ in high-Pi media at $30^{\circ} \mathrm{C}$ and split into either $200 \mathrm{~mL}$ of high-Pi or no-Pi media and grown for 2 hours. Formaldehyde (Sigma) was added to a final concentration of $1 \%(\mathrm{v} / \mathrm{v})$ to crosslink chromatin, and the reaction was allowed to proceed for 15 minutes. Glycine (Sigma) was then added to a final concentration of $125 \mathrm{mM}$ and incubated for $5 \mathrm{~min}$ utes to quench cross-linking. Cells were lysed by bead beating $(6 \times 2$ min on, 2 min off $)$ and chromatin was sheared to 300-600 bp fragments using a Misonix Sonicator 3000. Immunoprecipitation was performed with $100 \mathrm{uL}$ of Protein G Dynabeads (Invitrogen) coupled to $4 \mathrm{uL}$ of anti-Protein A antibody (Sigma). Protein concentrations were measured using a Bradford Assay (Bio-Rad). Following the generation of ChIP lysate three aliquots of $650 \mu \mathrm{g}$ soluble protein were subject to immunoprecipitation and pooled just prior to elution from the beads. Samples were processed following the Illumina HTS guidelines with libraries of 200-300 bp selected via $2 \%$ agarose DNA gels. Libraries were amplified by PCR and purity was determined using an Agilent High-Sensitivity DNA kit on an Agilent Bioanalyzer. Libraries were sequenced on an Illumina HighSeq 2000 and 50bp reads were aligned to the S. pombe $972 h^{-}$genome using ELAND. We obtained between 16 million and 54 million reads on average from our samples. Uniquely aligned reads were extended 80 bp from the read start site to cover the average length of insert as determined by the Agilent Bioanalyzer. Results for all of the ChIP-Seq experiments conducted in this study are available through NCBI-GEO [GEO:GSE39498]. 
To determine which of our enriched regions were actually attributable to a Pho7-TAP binding event we used a modified method from [48]. For each condition analyzed we set a lower threshold for peak discovery equal to the genomic average of reads per base. We set the upper threshold equal to the highest observed read count within the given sample. Using 380 equal increments between these two thresholds we defined peaks that were larger than 100 nucleotides and separated by at least 20 nucleotides. Peaks were compiled at the highest threshold at which they met those standards and full peaks were required to be at least 150 nucleotides distant from the nearest neighbor. Statistical analysis comparing sample enrichment to mock enrichment was performed in MATLAB using previously described methods [48]. Peaks used for subsequent analysis had a $\geq 2$-fold enrichment over the genome average and a pvalue $\leq 0.005$ compared to the mock sample.

To determine the likelihood that the genes determined from microarray analysis to be regulated by $\mathrm{Pi}$ and/or pho7 would also have promoters that contain at least one Pho7 binding site we utilized a hypergeometric test. In this case the $\mathrm{p}$-value is given by:

$$
P=1-\sum_{i=0}^{k-1} \frac{\left(\begin{array}{c}
M \\
i
\end{array}\right)\left(\begin{array}{c}
N-M \\
n-i
\end{array}\right)}{\left(\begin{array}{c}
N \\
i
\end{array}\right)}
$$

where $\mathrm{N}$ is the total number of genes probed by the microarray (5046), $\mathrm{M}$ is the number of peaks within -800 and $0 \mathrm{bp}$ of any start codon in no-Pi conditions (570), $\mathrm{n}$ is the full set of regulated genes ( 22 for Pi, 29 for $p h o 7^{+}$, and 7 for both), and $\mathrm{k}$ is the set of regulated genes with at least one Pho7 peak in the promoter (13 for Pi, 16 for $p h o 7^{+}$, and 6 for both).

\section{Western blotting with Pho7-TAP}

DP1, DP94, or DP114 Cells were grown to log-phase in high-Pi media at $30^{\circ} \mathrm{C}$. Following collection of a high-Pi sample, cells were washed three times in no-Pi media, transferred to no-Pi media, and grown for either 60 or 120 minutes. Cells were lysed by beadbeating in urea lysis buffer $\left(20 \mathrm{mM}\right.$ Tris- $\mathrm{HCl}, \mathrm{pH}$ 8.0, $50 \mathrm{mM} \mathrm{Na} 2 \mathrm{HPO}_{4}, 8 \mathrm{M}$ urea, and $1 \mathrm{mM}$ PMSF). Total protein was quantified on a Nanodrop 2000 (Thermo Scientific) using a BSA standard (Bio-Rad). Equal amounts of total protein for each sample (30 ug) were subjected to separation by SDS-PAGE and transferred to nitrocellulose. Immunoblotting was performed with rabbit IgG (1:1000, Jackson ImmunoResearch) followed by incubation with goat anti-rabbit-HRP (1:5000, Thermo Scientific). Blots were developed using the SuperSignal West Femto Chemiluminescent Substrate
(Thermo Scientific) and analyzed on an Alpha Innotech Gel Imagining System.

\section{pho $1^{+}$promoter deletion analysis}

Segments of the $p h o 1^{+}$promoter were amplified using PCR and cloned into a $y f^{+}$plasmid using homologous recombination [51] containing the selectable $\mathrm{ura}^{+}$marker, creating a $p h o 1^{+} p r-y f p^{+}$fusion. Plasmids were transformed into DP18, DP109, or DP111 backgrounds using lithium acetate and polyethylene glycol 8000. Cells were selected based on their ability to grow in EMM-ura media. Cells containing the various plasmids were grown to early log-phase $\left(\mathrm{OD}_{600} \sim 0.18\right)$ in high-Pi media (lacking uracil) at $30^{\circ} \mathrm{C}$, collected, washed twice in sterile water, and split into either high-Pi or no-Pi media (both lacking uracil). Cells were grown for 4 hours at $30^{\circ} \mathrm{C}$ and $100 \mathrm{uL}$ of $10 \%$ buffered formalin (Sigma) was added to $900 \mathrm{uL}$ culture. Fixation proceeded for 5 minutes at room temperature prior to washing: once with $0.1 \mathrm{M}$ potassium phosphate buffer $(\mathrm{pH}$ 8.5) and once with $1.2 \mathrm{M}$ sorbitol in $0.1 \mathrm{M}$ potassium phosphate buffer $(\mathrm{pH}$ 8.5). Cells were resuspended in $1.2 \mathrm{M}$ sorbitol, $0.1 \mathrm{M}$ potassium phosphate buffer $(\mathrm{pH}$ 8.5) and incubated overnight at $4^{\circ} \mathrm{C}$.

FACS counting with each sample was performed using a LSR II Analyzer (BD Biosciences). DP18, DP109, and DP111 cells lacking the $y f p^{+}$expression system were used to normalize forward-scatter, side-scatter, and autofluorescence for each experiment. 50,000 cells were counted for each experimental condition tested; cells with forward- and side-scatter values between 50,000150,000 and YFP expression $\geq$ mean autofluorescence were subject to further analysis. Three biological replicates were performed and the average YFP intensity for the replicates is reported $\pm \mathrm{SE}$.

\section{pho $7^{+}$regulation in additional stress response pathways}

For each individual stress response, initial cultures of DP1 and DP81 were grown in 90\%SD-10\%EMM media containing $10 \mathrm{mM} \mathrm{KH} \mathrm{KO}_{4}$ to early-log phase at $30^{\circ} \mathrm{C}$. Cells were collected, washed twice with autoclaved water, and split into the following conditions (all modifi-

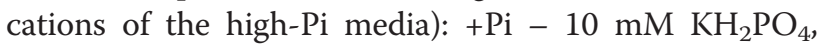
$-\mathrm{Pi}-0 \mathrm{mM} \mathrm{KH} \mathrm{PO}_{4},+\mathrm{Fe}-100$ uM Fe(III)Cl $\mathrm{Cl}_{3},-\mathrm{Fe}-$ 250 uM 2-2'-dipyridine (DIP) (Sigma), +Cu - $100 \mathrm{uM}$ $\mathrm{Cu}(\mathrm{II}) \mathrm{SO}_{4},-\mathrm{Cu}-100 \mathrm{uM}$ bathocuproine disulphonate (BCS) (Sigma), Osmotic Shift $-1.2 \mathrm{M} \mathrm{NaCl}$ instead of $0.1 \mathrm{M} \mathrm{NaCl}$, and Carbon Switch - 2\% glycerol/1\% ethanol (GE) instead of $2 \%$ glucose (G). Cells were grown for 2 hours and harvested as described above. Recovered RNA was converted into cDNA using the iScript cDNA synthesis kit (Bio-Rad) and subjected to RT-qPCR. Amplification of the $g p d 1^{+}, f i o 1^{+}, c t r 4^{+}, h x k 2^{+}$, and $p h o 1^{+}$ transcripts were measured for the three independent 
replicates and transcript abundance was normalized to $a c t 1^{+}$. Shown is the average \pm SE. Primers used in the RT-qPCR analysis can be found in Additional file 11.

Extracted RNA was also subjected to microarray analysis as detailed above. Expression from $p h o 7^{+}$cells in replete conditions was compared to that in stress conditions for each individual stressor to determine the base set of genes that respond in each given stress. The dependence upon $p h o 7^{+}$was determined by comparing the levels of induction in $p h o 7^{+}$cells in stress to induc-

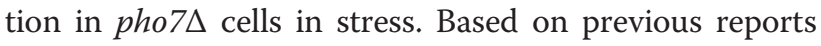
[27] the osmotic shift conditions were assayed $20 \mathrm{~min}$ utes post-shift to provide a more accurate measure of genes directly induced by osmotic pressure. Extraction of Cy3-Cy5 fluorescence intensity was performed using the GenePix 5.1 software and normalization was completed using the MATLAB bioinformatics toolbox. At least two independent biological replicates were performed for each of the conditions tested. For each of these arrays the two probes used to detect each ORF were averaged and treated as single data points with $\mathrm{p}$-values determined using a student's t-test with a one-tailed distribution against the null hypothesis in the MATLAB software. Thresholds were set at $\geq 1$.5-foldchange for all conditions with the exception of the $[-\mathrm{Cu} /+\mathrm{Cu}]$ and $[\mathrm{GE} / \mathrm{G}]$ arrays. For those conditions a significantly larger proportion of genes were induced, so we set the thresholds at $\geq[2 \sigma+$ median $] \log _{2}$ foldchange to ensure a similar sized cohort of analyzed genes. Genes passing the induction threshold also had to pass a $\mathrm{p}$-value threshold of $\leq 0.10$.

\section{Additional files}

Additional file 1: Figure S1. Temporal Dynamics of the Phosphate Starvation Response in S. pombe. (A) Line plot depicting the induction profile for genes displaying a fast (red) or slow (blue) response to phosphate starvation as measured by microarray analysis. Thresholds used to delineate the response time are described in the text. Induction was normalized to the initial sample pre-starvation $(t=0)$. (B) Average expression values for genes in the fast $(\mathbf{m})$ and slow $(\mathbf{O})$ response are shown \pm SD.

Additional file 2: Table S1. Microarray Results for Genes Identified in the Pi Starvation Time Course. Genes induced following two hours (fast response, bold) or four hours (slow response) of Pi depletion were tabulated and their normalized expression levels (to $t=0 \mathrm{~min}$ ) are shown. Induction thresholds for the fast response were set at $\geq 2 \sigma+$ median $\log _{2}$ fold change for each time point $\left(1.00 \log _{2}\right.$ fold change at 120 minutes, $1.24 \log _{2}$ fold change at 240 minutes). Genes above threshold at both 120 minutes and 240 minutes post-starvation were classified as the rapid response. Genes above only the 240-minute threshold were classified as the slow response.

Additional file 3: Table S2. Microarray Results for Genes Regulated by Pi Starvation, $p h o 7^{+}$, and/or $\operatorname{csk} 1^{+}$. Genes induced following Pi starvation, in $p h o 7^{+}$versus pho $7 \Delta$ cells during Pi starvation, or in csk $1 \Delta$ versus $\operatorname{csk} 1^{+}$ cells in non-stressed (high-Pi) conditions were tabulated and analyzed for orthologs in the S. cerevisiae PHO pathway. Induction thresholds were set at $\geq 1.8$ fold-change with a $p$-value $\leq 0.10$. Values shown are the average of two independent replicates.
Additional file 4: Figure S2. Global Pho7-TAP Enrichment During PiStarvation. Cells containing the tagged variant of Pho7 (Pho7-TAP) were grown in either high-Pi (blue) or starvation (red) media for 120 minutes prior to cross-linking and ChIP-Seq processing. Shown are the chromosomal enrichment profiles for Pho7 for the S. pombe genome. Reads were normalized to total counts for each chromosome.

Additional file 5: Table S3. Peak List for Pho7-TAP ChIP-Seq Data Set. Pho7-TAP peaks detected in both high-Pi (Hi) or no-Pi (No) conditions with maximum height $\geq 2 x$ genome average are given. Peak detection was performed against a mock sample lacking a TAP epitope as described in materials and methods.

Additional file 6: Figure S3. Pho7-TAP Protein Levels Remain Constant During Pi Starvation in $\operatorname{csk} 1^{+}$and $\operatorname{csk} 1 \Delta$ Backgrounds. $\operatorname{csk} 1^{+}$or $\operatorname{csk} 1 \Delta$ cells containing the tagged variant of Pho7 (Pho7-TAP) were grown in high-Pi (+Pi) media followed by Pi starvation for 60 or 120 minutes (-Pi). Western blot analysis reveals similar levels of the Pho7-TAP protein in all conditions as detected by rabbit lgG. The strain lacking the TAP tag is shown (Pho7) as a negative control. The Western analysis was completed in duplicate, shown is a representative blot.

Additional file 7: Figure S4. Global Pho7-TAP Enrichment in the

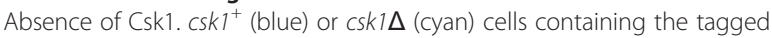
variant of Pho7 (Pho7-TAP) were grown in high-Pi media for 120 minutes prior to cross-linking and ChIP-Seq processing. Shown are the chromosomal enrichment profiles for Pho7 for the S. pombe genome. Reads were normalized to total counts for each chromosome.

Additional file 8: Figure S5. ChIP-Seq Binding Profiles for Pho7-TAP at Non-PHO Promoters. Shown are ChIP-Seq profiles for the genes identified in Figure 6. As previously described, wild-type cells containing Pho7-TAP were grown in either high-Pi (blue) or no-Pi (red) conditions and ChIPSeq libraries were prepared from purified DNA. For comparison, the ChIPSeq signal from mock (black) cells grown in no-Pi and cski $\Delta$ (cyan) cells incubated in high-Pi is included. The gene product of interest is plotted based on transcript direction with the plus (+) strand above and the minus (-) strand below. Reads were normalized as described in the text and the location within the genome is plotted on the $x$-axis.

Additional file 9: Table S4. Gene Ontology Enrichment for Pho7 Dependent Genes Identified via Microarray and ChIP-Seq Analysis. Genes possessing a pho $7^{+}$-dependency independent of Pi availability were cross-referenced with the Pho7-TAP peak list to identify pho $7^{+}$-regulated genes with promoters enriched by Pho7-TAP. Thresholds for induction and peak enrichment are described in materials and methods. The gene set was processed through the AmiGO toolkit [34,52-55] and GO terms containing at least 3 genes with $p$-values $\leq 0.01$ are displayed.

Additional file 10: Table S5. Microarray Results for $p h o 7^{+}$-Dependent Genes Regulated by Phosphate, Iron, Copper, Osmotic, and/or Carbon Switching Stress. pho $7^{+}$genes passing the thresholds (described in materials and methods) for each stress condition assayed were tabulated with the corresponding fold-change $\left(\log _{2}\right)$ and $p$-values (based on at least two independent biological replicates). Genes regulated in multiple stress conditions by pho $7^{+}$are indicated in bold. All comparisons between stressed and non-stressed conditions (e.g., [-Pi/+Pi]) were done in a pho7+ background. Genes passing the thresholds for each array condition have fold-change $\left(\mathrm{Log}_{2}\right)$ values indicated in bold. $+\mathrm{Pi}: 10 \mathrm{mM}$ H2KPO4, -Pi: 0 mM H2KPO4, +Fe: 100 uM Fe(III)Cl3, -Fe: 250 uM DIP, +Cu: 100 uM Cu(II)SO4, -Cu: 100 uM BCS, 1.2M: 1.2M NaCl, 0.1M: 0.1M NaCl, G: $2 \%$ glucose, GE: $2 \%$ glycerol, $1 \%$ ethanol.

Additional file 11: Table S6. All primers utilized in this manuscript are listed with their primer ID, nucleotide sequence, and experimental purpose.

\section{Competing interests}

The authors declare that they have no competing interests.

\section{Authors' contributions}

The manuscript was written by ICO. Microarrays, ChIP-Seq, and FACS quantization were performed by ICO. DDW provided S. pombe strains. MP prepared the pho $1^{+}$pr-yfp ${ }^{+}$constructs. Computational analysis was performed by ICO using modified methods from previously published sources $[7,46,48]$. 
EKO and DDW provided supervision throughout and EKO and DDW are the corresponding authors. All authors read and approved the final manuscript.

\section{Acknowledgements}

The authors wish to thank C. Daly and J. Zhang for help with Illumina sequencing, V. Vijayan, X. Zhou, and A. Hansen for assistance with MATLAB and statistical analysis, and all members of the O'Shea lab for thoughtful discussion and commentary. This work was supported by NSF-MCB-1121714, Villanova University and the Howard Hughes Medical Institute.

\section{Author details}

${ }^{1}$ Howard Hughes Medical Institute, Faculty of Arts and Sciences, Center for Systems Biology, Northwest Labs, Harvard University, 52 Oxford Street, Cambridge, MA 02138, USA. ²Department of Molecular and Cellular Biology, Harvard University, Faculty of Arts and Sciences, Center for Systems Biology, Northwest Labs, 52 Oxford Street, Cambridge, MA 02138, USA. ${ }^{3}$ Department of Chemistry and Chemical Biology, Harvard University, Faculty of Arts and Sciences, Center for Systems Biology, Northwest Labs, 52 Oxford Street, Cambridge, MA 02138, USA. ${ }^{4}$ Department of Biology, Villanova University, 800 Lancaster Ave, Villanova, PA 19085, USA.

Received: 22 August 2012 Accepted: 6 December 2012 Published: 12 December 2012

\section{References}

1. Abel S: Phosphate sensing in root development. Curr Opin Plant Biol 2011, 14:303-309.

2. Bergwitz C, Juppner H: Phosphate sensing. Adv Chronic Kidney Dis 2011, 18:132-144.

3. Wykoff DD, O'Shea EK: Phosphate transport and sensing in Saccharomyces cerevisiae. Genetics 2001, 159:1491-1499.

4. Doerner $P$ : Phosphate starvation signaling: a threesome controls systemic P(i) homeostasis. Curr Opin Plant Biol 2008, 11:536-540.

5. Hulett FM: The signal-transduction network for Pho regulation in Bacillus subtilis. Mol Microbiol 1996, 19:933-939.

6. Ogawa N, DeRisi J, Brown PO: New components of a system for phosphate accumulation and polyphosphate metabolism in Saccharomyces cerevisiae revealed by genomic expression analysis. Mol Biol Cell 2000, 11:4309-4321.

7. Zhou X, O'Shea EK: Integrated approaches reveal determinants of genome-wide binding and function of the transcription factor Pho4. Mol Cell 2011, 42:826-836.

8. Springer M, Wykoff DD, Miller N, O'Shea EK: Partially phosphorylated Pho4 activates transcription of a subset of phosphate-responsive genes. PLOS Biol 2003, 1:E28

9. Lenburg ME, O'Shea EK: Signaling phosphate starvation. Trends Biochem Sci 1996, 21:383-387.

10. Oshima Y: The phosphatase system in Saccharomyces cerevisiae. Genes Genet Syst 1997, 72:323-334.

11. Mouillon JM, Persson BL: New aspects on phosphate sensing and signalling in Saccharomyces cerevisiae. FEMS Yeast Res 2006, 6:171-176.

12. Kaffman A, Herskowitz I, Tjian R, O'Shea EK: Phosphorylation of the transcription factor $\mathrm{PHO} 4$ by a cyclin-CDK complex, $\mathrm{PHO} 80-\mathrm{PHO} 85$. Science 1994, 263:1153-1156.

13. O'Neill EM, Kaffman A, Jolly ER, O'Shea EK: Regulation of PHO4 nuclear localization by the PHO80-PHO85 cyclin-CDK complex. Science 1996, 271:209-212.

14. Kaffman A, Rank NM, O'Neill EM, Huang LS, O'Shea EK: The receptor Msn5 exports the phosphorylated transcription factor Pho4 out of the nucleus. Nature 1998, 396:482-486.

15. Komeili A, O'Shea EK: Roles of phosphorylation sites in regulating activity of the transcription factor Pho4. Science 1999, 284:977-980.

16. Lee YS, Mulugu S, York JD, O'Shea EK: Regulation of a cyclin-CDK-CDK inhibitor complex by inositol pyrophosphates. Science 2007, 316:109-112.

17. Lee YS, Huang K, Quiocho FA, O'Shea EK: Molecular basis of cyclin-CDKCKI regulation by reversible binding of an inositol pyrophosphate. Nat Chem Biol 2008, 4:25-32.

18. Kaffman A, Rank NM, O'Shea EK: Phosphorylation regulates association of the transcription factor Pho4 with its import receptor Pse1/Kap121. Genes Dev 1998, 12:2673-2683.
19. Schmidt G, Bartsch G, Laumont MC, Herman T, Liss M: Acid phosphatase of bakers' yeast: an enzyme of the external cell surface. Biochemistry 1963, 2:126-131

20. Vogel $K$, Horz W, Hinnen A: The two positively acting regulatory proteins $\mathrm{PHO} 2$ and $\mathrm{PHO} 4$ physically interact with $\mathrm{PHO} 5$ upstream activation regions. Mol Cell Biol 1989, 9:2050-2057.

21. Maerkl SJ, Quake SR: A systems approach to measuring the binding energy landscapes of transcription factors. Science 2007, 315:233-237.

22. Turunen $\mathrm{O}$, Seelke $\mathrm{R}$, Macosko J: In silico evidence for functional specialization after genome duplication in yeast. FEMS Yeast Res 2009, 9:16-31.

23. Tanaka K, Okayama H: A pcl-like cyclin activates the Res2p-Cdc10p cell cycle "start" transcriptional factor complex in fission yeast. Mol Biol Cell 2000, 11:2845-2862.

24. Henry TC, Power JE, Kerwin CL, Mohammed A, Weissman JS, Cameron DM Wykoff DD: Systematic screen of Schizosaccharomyces pombe deletion collection uncovers parallel evolution of the phosphate signal transduction pathway in yeasts. Eukaryot Cell 2011, 10:198-206.

25. MacPherson S, Larochelle M, Turcotte B: A fungal family of transcriptional regulators: the zinc cluster proteins. Microbiol Mol Biol Rev 2006, 70:583-604.

26. Hermand D, Pihlak A, Westerling T, Damagnez V, Vandenhaute J, Cottarel G, Makela TP: Fission yeast Csk1 is a CAK-activating kinase (CAKAK). EMBO J 1998, 17:7230-7238.

27. Chen D, Toone WM, Mata J, Lyne R, Burns G, Kivinen K, Brazma A, Jones N, Bahler J: Global transcriptional responses of fission yeast to environmental stress. Mol Biol Cell 2003, 14:214-229.

28. Gasch AP, Spellman PT, Kao CM, Carmel-Harel O, Eisen MB, Storz G, Botstein D, Brown PO: Genomic expression programs in the response of yeast cells to environmental changes. Mol Biol Cell 2000, 11:4241-4257.

29. Hao N, O'Shea EK: Signal-dependent dynamics of transcription factor translocation controls gene expression. Nat Struct Mol Biol 2011, 19:31-39.

30. Maundrell K, Nurse P, Schonholzer F, Schweingruber ME: Cloning and characterization of two genes restoring acid phosphatase activity in pho1- mutants of Schizosaccharomyces pombe. Gene 1985, 39:223-230.

31. Sonnhammer EL, Eddy SR, Durbin R: Pfam: a comprehensive database of protein domain families based on seed alignments. Proteins 1997, 28:405-420.

32. Punta M, Coggill PC, Eberhardt RY, Mistry J, Tate J, Boursnell C, Pang N, Forslund K, Ceric G, Clements J, et al: The Pfam protein families database. Nucleic Acids Res 2012, 40:D290-D301.

33. Schweingruber ME, Edenharter E, Zurlinden A, Stockmaier KM: Regulation of pho1-encoded acid phosphatase of Schizosaccharomyces pombe by adenine and phosphate. Curr Genet 1992, 22:289-292.

34. Carbon S, Ireland A, Mungall CJ, Shu S, Marshall B, Lewis S: AmiGO: online access to ontology and annotation data. Bioinformatics 2009, 25:288-289.

35. Ohmiya R, Yamada H, Nakashima K, Aiba H, Mizuno T: Osmoregulation of fission yeast: cloning of two distinct genes encoding glycerol-3phosphate dehydrogenase, one of which is responsible for osmotolerance for growth. Mol Microbiol 1995, 18:963-973.

36. Petit T, Blazquez MA, Gancedo C: Schizosaccharomyces pombe possesses an unusual and a conventional hexokinase: biochemical and molecular characterization of both hexokinases. FEBS Lett 1996, 378:185-189.

37. Askwith C, Kaplan J: An oxidase-permease-based iron transport system in Schizosaccharomyces pombe and its expression in Saccharomyces cerevisiae. J Biol Chem 1997, 272:401-405.

38. Pelletier B, Beaudoin J, Mukai Y, Labbe S: Fep1, an iron sensor regulating iron transporter gene expression in Schizosaccharomyces pombe. J Biol Chem 2002, 277:22950-22958.

39. Labbe S, Pena MM, Fernandes AR, Thiele DJ: A copper-sensing transcription factor regulates iron uptake genes in Schizosaccharomyces pombe. J Biol Chem 1999, 274:36252-36260.

40. Kim DU, Hayles J, Kim D, Wood V, Park HO, Won M, Yoo HS, Duhig T, Nam M, Palmer G, et al: Analysis of a genome-wide set of gene deletions in the fission yeast Schizosaccharomyces pombe. Nat Biotechnol 2010, 28:617-623.

41. Pei Y, Du H, Singer J, Stamour C, Granitto S, Shuman S, Fisher RP: Cyclin-dependent kinase 9 (Cdk9) of fission yeast is activated by the CDK-activating kinase Csk1, overlaps functionally with the TFIIHassociated kinase Mcs6, and associates with the mRNA cap methyltransferase Pcm1 in vivo. Mol Cell Biol 2006, 26:777-788. 
42. Garriga J, Grana X: Cellular control of gene expression by T-type cyclin/ CDK9 complexes. Gene 2004, 337:15-23.

43. Martinez-Pastor MT, Marchler G, Schuller C, Marchler-Bauer A, Ruis H, Estruch F: The Saccharomyces cerevisiae zinc finger proteins Msn2p and Msn4p are required for transcriptional induction through the stress response element (STRE). EMBO J 1996, 15:2227-2235.

44. Gorner W, Durchschlag E, Martinez-Pastor MT, Estruch F, Ammerer G, Hamilton B, Ruis $\mathrm{H}$, Schuller $\mathrm{C}$ : Nuclear localization of the $\mathrm{C} 2 \mathrm{H} 2$ zinc finger protein Msn2p is regulated by stress and protein kinase A activity. Genes Dev 1998, 12:586-597.

45. Forsburg SL, Rhind N: Basic methods for fission yeast. Yeast 2006, 23:173-183.

46. Vijayan V, Zuzow R, O'Shea EK: Oscillations in supercoiling drive circadian gene expression in cyanobacteria. Proc Natl Acad Sci USA 2009, 106:22564-22568

47. Eisen MB, Spellman PT, Brown PO, Botstein D: Cluster analysis and display of genome-wide expression patterns. Proc Natl Acad Sci USA 1998, 95:14863-14868.

48. Vijayan $\mathrm{V}$, Jain $\mathbb{H}$, O'Shea EK: A high resolution map of a cyanobacterial transcriptome. Genome Biol 2011, 12:R47.

49. Lam FH, Steger DJ, O'Shea EK: Chromatin decouples promoter threshold from dynamic range. Nature 2008, 453:246-250

50. Monahan BJ, Villen J, Marguerat S, Bahler J, Gygi SP, Winston F: Fission yeast SWI/SNF and RSC complexes show compositional and functional differences from budding yeast. Nat Struct Mol Biol 2008, 15:873-880.

51. Chino A, Watanabe K, Moriya H: Plasmid construction using recombination activity in the fission yeast Schizosaccharomyces pombe. PLoS One 2010, 5:e9652.

52. Wapinski I, Pfeffer A, Friedman N, Regev A: Natural history and evolutionary principles of gene duplication in fungi. Nature 2007, 449:54-61.

53. Hertz-Fowler C, Peacock CS, Wood V, Aslett M, Kerhornou A, Mooney P, Tivey A, Berriman M, Hall N, Rutherford K, et al: GeneDB: a resource for prokaryotic and eukaryotic organisms. Nucleic Acids Res 2004, 32:D339-D343.

54. Ashburner M, Ball CA, Blake JA, Botstein D, Butler H, Cherry JM, Davis AP, Dolinski K, Dwight SS, Eppig JT, et al: Gene ontology: tool for the unification of biology. The Gene Ontology Consortium. Nat Genet 2000, 25:25-29.

55. Boyle El, Weng S, Gollub J, Jin H, Botstein D, Cherry JM, Sherlock G: GO: TermFinder-open source software for accessing Gene Ontology information and finding significantly enriched Gene Ontology terms associated with a list of genes. Bioinformatics 2004, 20:3710-3715.

doi:10.1186/1471-2164-13-697

Cite this article as: Carter-O'Connell et al: Genome-Wide Characterization of the Phosphate Starvation Response in Schizosaccharomyces pombe. BMC Genomics 2012 13:697.

\section{Submit your next manuscript to BioMed Central and take full advantage of:}

- Convenient online submission

- Thorough peer review

- No space constraints or color figure charges

- Immediate publication on acceptance

- Inclusion in PubMed, CAS, Scopus and Google Scholar

- Research which is freely available for redistribution 